\title{
From Boolean Algebra to PLC Logic
}

\section{IVANA ZUBAC \& SNJEZANA REZIC}

Abstract: Boolean algebra is a suitable tool that enable the conversion of control tasks into a PLC program. Objective of this paper is to present how Boolean equations can be converted into PLC logic, or logic and switching circuits. This paper briefly discuss on the basic needs of logic synthesis and also considers PLC design regarding development of the control program. An example is given to ilustrate obtain Boolean equations from logical syntesis and transformation equations using the axioms of Boolean algebra. After simplification the equation can be converted into ladder logic or a circuit diagram.

Key words: Boolean function, Combinatorial logic, Digital circuit, Switching circuit, Ladder logic.
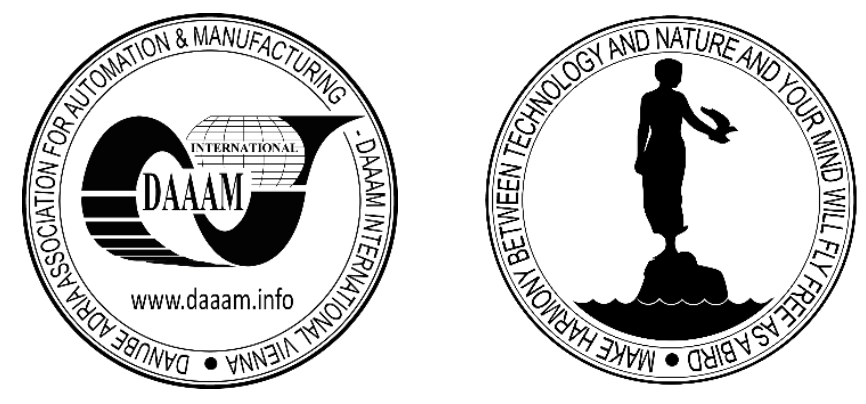

Authors' data: *Ivana Zubac \& Snjezana Rezic, University of Mostar, Faculty of Mechanical Engineering, Computing and Electrical Engineering, Matice hrvatske bb, 88000Mostar, BA, *ivana.zubac@ fsre.sum.ba

This Publication has to be referred as: Zubac, I[vana] \& Rezic, S[njezana] (2020). From Boolean Algebra to PLC Logic, Chapter 20 in DAAAM International Scientific Book 2020, pp.237-244, B. Katalinic (Ed.), Published by DAAAM International, ISBN 978-3-902734-27-3, ISSN 1726-9687, Vienna, Austria

DOI: $10.2507 /$ daaam.scibook.2020.20 


\section{Introduction}

PLC can be explained as a digital device that uses a programmable memory to store direction and to accomplish functions such as counting, logic sequencing, arithmetic and timing in order to control processes. It can receive information from sensors, actuators or other PLC logic input and output logic signal processed. Ladder logic is the main programming method used for PLCs. Boolean algebra or switching algebra is the basis of digital control systems, such as PLC.

The process of converting control objectives into a ladder logic diagram requires knowledge of Boolean algebra. Boolean algebra provides the tools needed to analyse and design these systems. Reference [1] proposed a method to design a Ladder Diagram based on a logic design approach. Wakerly J.F [2] describes digital principals and the relations of control elements, especially logic elements based on Boolean algebra. In paper $[3,4]$ authors explain process of converting from a ladder diagram to a native code directly. The primary step of PLC control model is to the establishment of PLC model, such as establish a model from Function Charts, Logic diagram, Boolean equation, etc. [5,6]. In this paper we describe how design ideas can be converted to Boolean equations directly and then converted into ladder logic, or a switching circuit.

\section{Boolean Algebra}

Boolean algebra is a mathematical structure, introduced by George Boole in 1847. as a tool for the mathematical analysis of logic. It is defined using the meet and join operators instead of the usual addition and multiplication operators. Explicitly, a Boolean algebra is the partial order on subsets defined by inclusion [7], that is the Boolean algebra $b(A)$ of a set $A$ is the set of subsets of $A$ that can be obtained by means of a finite number of the set operations union (OR) denote by $a \mathrm{~V}$ $b$, intersection (AND) denote by $a \wedge b$, and complementation (NOT) denote by $\neg a$ or $\bar{a}$, Table 1. A Boolean algebra also forms a lattice, and each of the elements of $b(A)$ is called a Boolean function. There are $2^{2^{n}}$ Boolean functions in a Boolean algebra of order $n$.

\begin{tabular}{|c|c|c|c|}
\hline $\boldsymbol{a}$ & $\boldsymbol{b}$ & $\boldsymbol{a} \wedge \boldsymbol{b}$ & $\boldsymbol{a} \vee \boldsymbol{b}$ \\
\hline 1 & 1 & 1 & 1 \\
\hline 1 & 0 & 0 & 1 \\
\hline 0 & 1 & 0 & 1 \\
\hline 0 & 0 & 0 & 0 \\
\hline
\end{tabular}

\begin{tabular}{|l|l|}
\hline $\mathrm{a}$ & $\neg a(\bar{a})$ \\
\hline 1 & 0 \\
\hline 0 & 1 \\
\hline
\end{tabular}

Tab. 1. Operation of Boolean algebra. 
At the beginning it was not used for practical purposes. In 1938, Shannon proved that a two-valued Boolean algebra who can describe the operation of two-valued electrical switching circuits. In 1950.s, the switching theory was extended to include sequential systems, i.e., systems whose outputs depend not only on the inputs but also on the previous states of the system [8]. Digital electronic computers and other digital systems were developed based on these advances in theory. In this days, digital electronic systems are widely used, and the corresponding theories are studied in mathematics, computer science, and electrical engineering. A digital system is defined as a system whose inputs and outputs are discrete quantities. These quantities are normally represented by binary variables, whit two possible values: 0 and 1 (also known as false and true). A special case of Boolean algebra is often called switching algebra because of its direct physical analogy with switching circuits or logical algebra. A function that accepts binary inputs and provides binary outputs is called a binary function. A binary function can be defined by a corresponding truth table that provides the output for all possible combinations of values of the input variables. The equations can be manipulated using the basic axioms of Boolean $(\forall a, b, c \in A)$ shown in Table 2.

\begin{tabular}{|l|c|}
\hline Axiom & Description \\
\hline Idempotent & $a \wedge a=a \vee a=a$ \\
\hline Commutative & $\begin{array}{r}a \wedge b=b \wedge a \\
a \vee b=b \vee a\end{array}$ \\
\hline Asociative & $a \wedge(b \wedge c)=(a \wedge b) \wedge c$ \\
& $a \vee(b \vee c)=(a \vee b) \vee c$ \\
\hline Distributive & $a \wedge(b \vee c)=(a \wedge b) \vee(b \wedge c)$ \\
& $a \vee(b \wedge c)=(a \vee b) \wedge(a \vee c)$ \\
\hline Identity & $a \vee 0=a, a \wedge 1=a$ \\
\hline Complement & $a \wedge \bar{a}=0$ \\
& $a \vee \bar{a}=1$ \\
\hline De Morgan & $\overline{a \vee b}=\bar{a} \wedge \bar{b}$ \\
\hline & $a \wedge b=\bar{a} \vee \bar{b}$ \\
\hline
\end{tabular}

Tab. 2. Axioms of Boolean algebra.

Whit interchange AND and OR operators, as well as all Universal, and Null sets, the resulting equation is equivalent original. This is known as the principle of duality. Boolean algebra is crucial to the understanding to logic design and switching circuit to timing analysis and PLC programming. A relationships between Boolean algebra, logic circuits, relay circuits and Ladder diagram are prerequisite for learning how to design and implement an control systems using PLC. Boolean algebra is the mathematical 
basis for logic design and establishes the means by which a task's defining rules are represented digitally. It is the best way for defines algorithmic steps, and the progression between steps is enabled by logic design. Mathematical symbolism, used in practical, technically oriented Boolean algebra, is not identical with notations used in theoretical mathematics. There is, however, unambiguous correspondence between the corresponding notation and the possibility of transcription is very easy.

\section{From Boolean Algebra to Ladder Logic}

The aim of this paper is explain the relationships between Boolean algebra and ladder diagrams. Table 3. shows relationship between Boolean logic, circuit diagram and ladder logic, which are necesssary for transformation between them.

\begin{tabular}{|c|c|c|c|c|c|}
\hline $\begin{array}{l}\text { Descripti } \\
\text { on }\end{array}$ & $\begin{array}{l}\text { Algebraic } \\
\text { equation } \\
\text { Truth tab }\end{array}$ & and & Logic simbol & $\begin{array}{l}\text { Circuits } \\
\text { symbol }\end{array}$ & Ladder symbol \\
\hline$A N D$ & $Y=A$ & & & & \\
\hline & \begin{tabular}{|l|l|}
$A$ & $B$ \\
\end{tabular} & $Y$ & & & \\
\hline & \begin{tabular}{l|l}
0 & 0
\end{tabular} & 0 & & & \\
\hline & \begin{tabular}{l|l}
0 & 1 \\
\end{tabular} & 0 & & & \\
\hline & \begin{tabular}{l|l}
1 & 0 \\
\end{tabular} & 0 & & & \\
\hline & \begin{tabular}{|l|l|}
1 & 1 \\
\end{tabular} & 1 & & & \\
\hline$O R$ & $Y=A-1$ & $+B$ & & & \\
\hline & \begin{tabular}{l|l|}
$A$ & $B$ \\
\end{tabular} & $Y$ & & & \\
\hline & \begin{tabular}{l|l|}
0 & 0 \\
\end{tabular} & 0 & & & \\
\hline & \begin{tabular}{l|l}
0 & 1 \\
\end{tabular} & 1 & & & \\
\hline & \begin{tabular}{l|l}
1 & 0 \\
\end{tabular} & 1 & & & \\
\hline & \begin{tabular}{l|l|}
1 & 1 \\
\end{tabular} & 1 & & & \\
\hline NOT & $Y=1$ & & & & $H^{A}$ \\
\hline & \begin{tabular}{|l|l|}
$A$ & $Y$ \\
\end{tabular} & & & & \\
\hline & \begin{tabular}{l|l}
0 & 1
\end{tabular} & & & 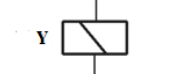 & \\
\hline & \begin{tabular}{l|l}
1 & 0
\end{tabular} & & & & \\
\hline NAND & $Y=\bar{A}$ & & & & $L^{A} L^{B}$ \\
\hline & \begin{tabular}{|l|l|}
$A$ & $B$ \\
\end{tabular} & $Y$ & & & \\
\hline & \begin{tabular}{l|l|}
0 & 0 \\
\end{tabular} & 1 & & & \\
\hline & \begin{tabular}{l|l|}
0 & 1 \\
\end{tabular} & 1 & & 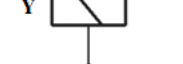 & \\
\hline & \begin{tabular}{l|l}
1 & 0 \\
\end{tabular} & 1 & & & \\
\hline & \begin{tabular}{l|l}
1 & 1 \\
\end{tabular} & 0 & & & \\
\hline
\end{tabular}




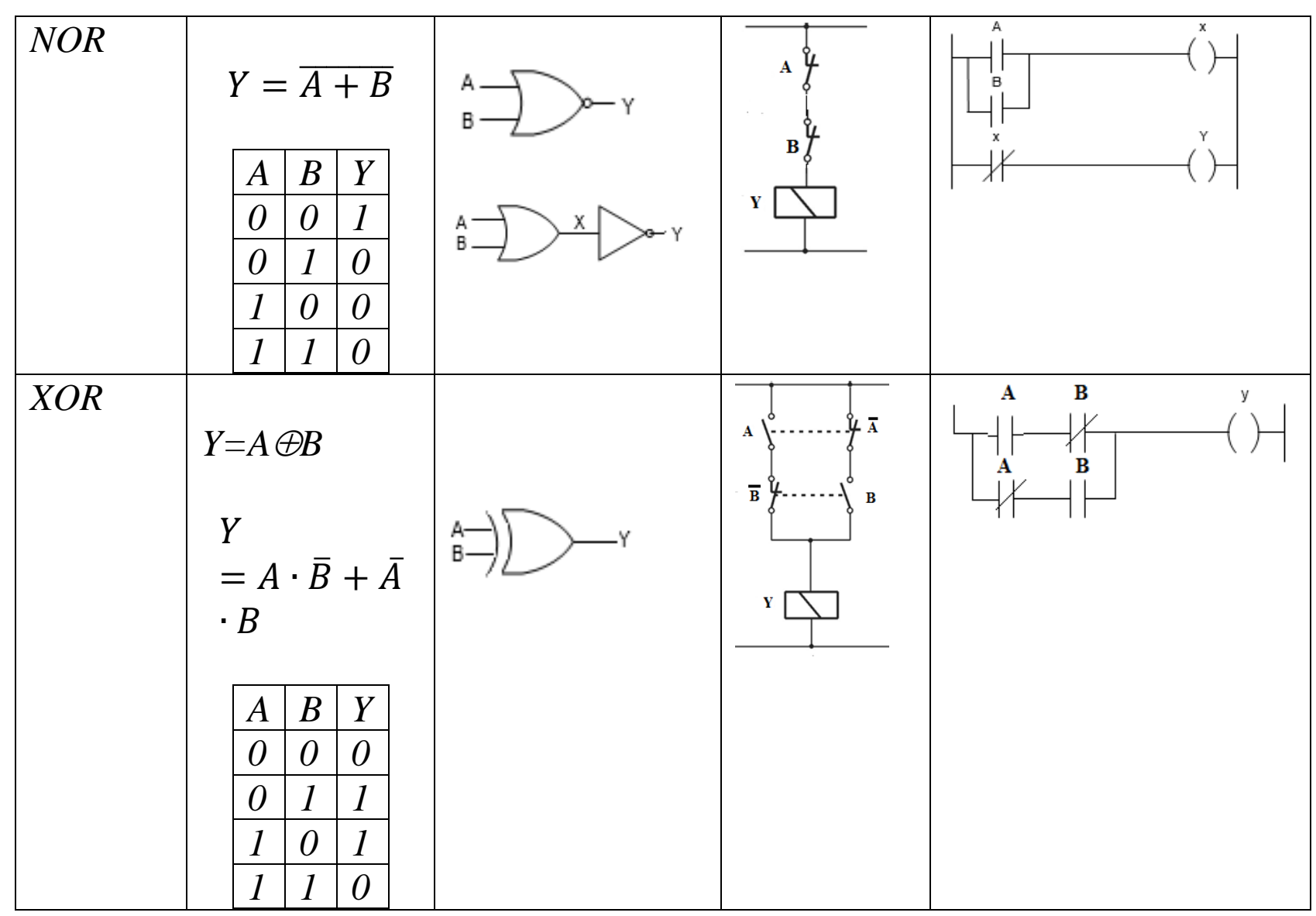

Tab. 3. Relationship between Boolean logic, circuit diagram and ladder logic.

\subsection{Practical example - Process Description}

If we can describe how a controller should work in words, we can often convert it directly to a Boolean equation. In the example a process descrip $\neg$ tion is given first. In this case it is written out in a sentence first, and then converted to a Boolean expression. The Boolean expression may then be converted to a desired form. The cutting device is supplied with workpieces on three sides (1). The pneumatic cylinder cuts a hole in the workpiece, when at least two of the three signals from the sensor are activated the cylinder returns to the starting position after ejecting the workpiece.

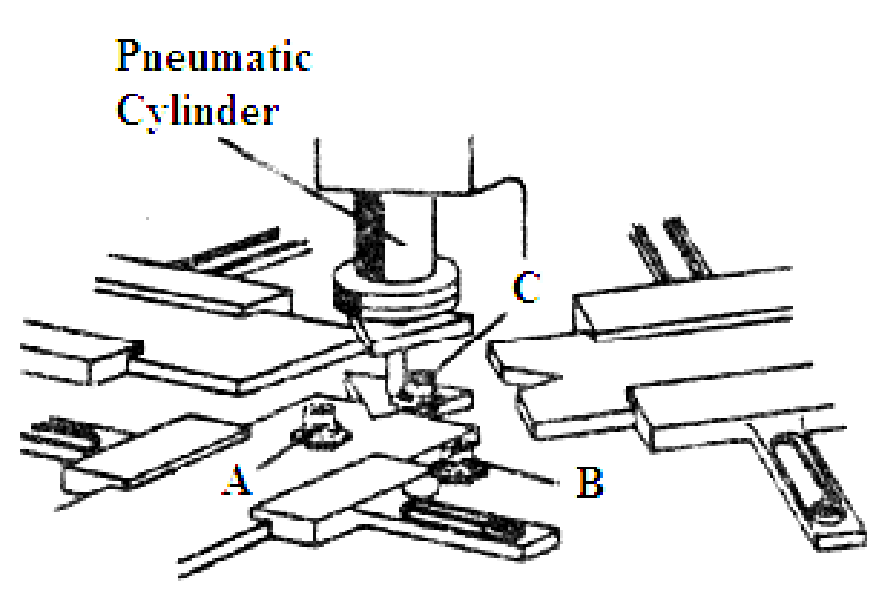

Fig. 1. Schematic representation of the process cuts. 
The next step is to define the controller equation. In this case the controller has 3 different inputs, and a single output, so a truth table is a reasonable approach to formaliz $\neg$ ing the system. A truth table is a complete enumeration of all possible combinations of input values, each one with its associated output value. When used to describe an existing circuit, output values are either 0 or 1 . We obtain the truth table 4.

Of the eight possible combinations of process inputs, only four lead to process condiations. A Boolean equation can then be written using the truth table in Table 4 . A Boolean equation is formed with binary variables, the binary operators AND and OR and the unary operator NOT. The Boolean algebraic expression is

$$
Y_{1}=\bar{A} B C+A \bar{B} C+A B \bar{C}+A B C
$$

\begin{tabular}{|l|l|l|l|l|}
\hline \multirow{2}{*}{ No. } & \multicolumn{3}{l}{ Sensor signal } & \multicolumn{2}{l|}{ Pneumatic cylinder } \\
\cline { 2 - 5 } & A & B & C & $\mathbf{Y}_{\mathbf{1}}$ \\
\hline 0 & 0 & 0 & 0 & 0 \\
\hline 1 & 0 & 0 & 1 & 0 \\
\hline 2 & 0 & 1 & 0 & 0 \\
\hline 3 & 0 & 1 & 1 & 1 \\
\hline 4 & 1 & 0 & 0 & 0 \\
\hline 5 & 1 & 0 & 1 & 1 \\
\hline 6 & 1 & 1 & 0 & 1 \\
\hline 7 & 1 & 1 & 1 & 1 \\
\hline
\end{tabular}

Tab. 4. Truth table for process.

The output Y1 (Boolean function) is equal to the sum of all rows that gives a value of 1.A Boolean function may be expressed algebraically from a given truth table by forming a midterm for each combination of variable that produce a 1 and taken the OR of those terms. After the equation has been written, it is sim $\neg$ plified.

Simplification using Boolean axiom (algebraic manipulation) can be done as follows:

$$
\begin{gathered}
Y_{1}=\bar{A} B C+A B C+A \bar{B} C+A B C+A B \bar{C}+A B C \\
=(\bar{A}+A) B C+(\bar{B}+B) A C+(\bar{C}+C) A B= \\
=B C+A C+A B=A B+A C+B C
\end{gathered}
$$

\subsection{Karnaugh map}

The Karnaugh map is easier than algebraic minimization because we just have to recognize patterns of $1 \mathrm{~s}$ in the map instead of using the algebraic manipulations. 
$\mathrm{AB}$

$\mathrm{C}$

\begin{tabular}{|l|l|l|l|l|}
\hline & 00 & 01 & 11 & 10 \\
\hline 0 & & &,$\hat{1}$ & \\
\hline 1 & & $(1, \ldots)$ & 1 & \\
\hline & & & & \\
\hline
\end{tabular}

Tab. 5. Karnaugh map.

Minimal algebraic equation is:

$$
Y_{1}=A B+A C+B C
$$

After simplification Boolean equation can be converted to ladder logic or switch circuits as shown on (3) and (4). Using relationship defined in Table 3. we can start design logic, switching or ladder diagram of the process.

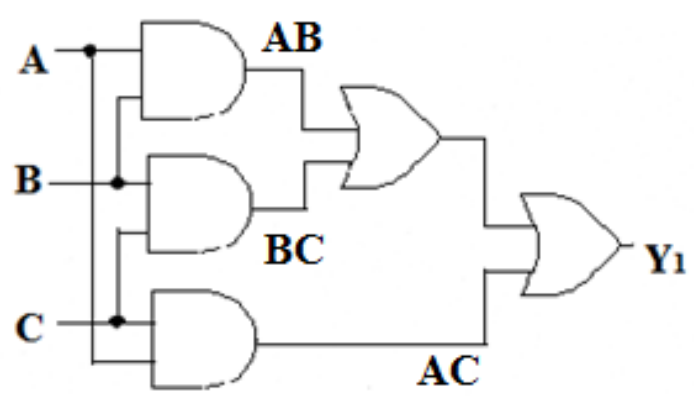

Fig. 2. Schematic representation of the process cuts.

A combinational circuit diagram a specific information processing operation fully specified logically by a set of Boolean functions.

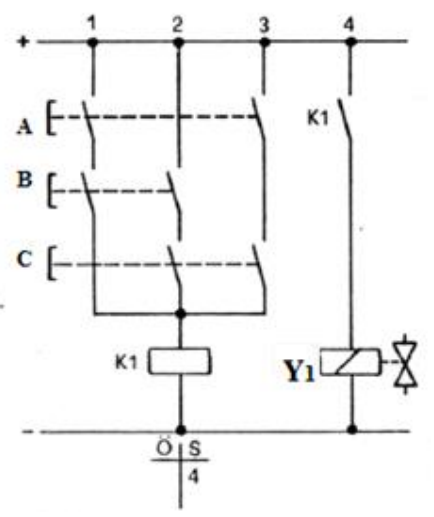

Fig. 3. Circuit diagrams of the cutting device.

Generating Boolean equations to implement a desired logic function is a necessary step before a circuit can be implemented. Ladder logic diagram is shown on figure 5 . 


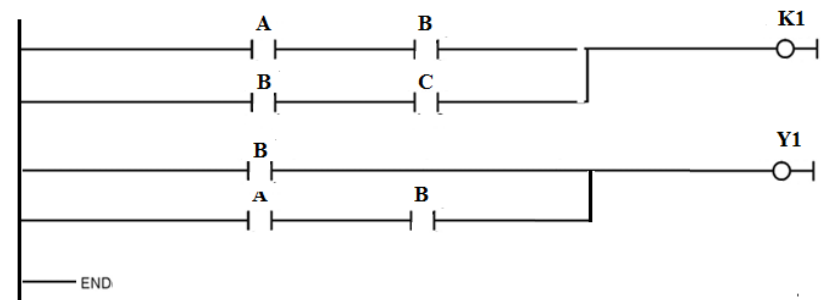

Fig. 4. Circuit diagrams of the cutting device.

From this example it is seen how the control model can be developed from the Boolean equations, but also that the reverse procedure is possible.

\section{Conclusion}

In this paper, a method to design an Ladder diagram, circuit diagram from Boolean logic is presented with typical logic operation and equation. Design ideas can be converted to Boolean equations on different way (using thrue table axioms, Karnaugh maps, logic design...). The equation can be manipulated using the axioms of Boolean algebra. After simplification the equation can be converted back into ladder logic or a circuit diagram. This paper involved the design activity on both algebraic analysis and Ladder diagram development of PLC in control of any process. The design on circuitry system is involved for connecting between input and output system. By using this technique, next step will be design of PLC control model using complex gates forms.

\section{References}

Bryan, L. A.; Bryan, E. A. (2008). Programmable Controllers Theory And Implementation, Industrial Text Company Publication, Atlanta, Georgia

Jack, H. (2005). Automating Manufacturing Systems with PLCs, Free Software Foundation

Selvaraj, K. (2010). Development of a "Programmable Logic Controller Circuitry" for Optimal Power Distribution in a Manufacturing Industries. Journal of Computer Science (6), 250-252.

Ismail, M. H. (2008). Rain Water PLC Based Detector and Valve Switcher, Project paper, Universiti Malaysia Pahang

Kim, H. S.; Kwon, W. H.; \& Chang, N. (1999). A translation method for ladder diagram with application to a manufacturing process, Proceedings of International Conference on Robotics and Automation, Vol. 1, 793-798.

Cui, X. L.; Zhou, Z. C. (2000). The algorithm of transformation between the ladder language and the sentence table language of PLC, Microelectronics and Computer, China, Vol. 1 (17), 26-30.

Weisstein, E. W. (1999). CRC Concise Encyclopedia of Mathematics, CRC Press, ISBN 1584883472, Boca Raton, Florida

Bourouis, A.; Belattar, B. (2012). Towards a metamodel for extended queuing networks, IJSIMM 11 (3), 117-128 